\title{
Resonance Scattering Phase Shifts in a 2-d Lattice Model *
}

\author{
C.R. Gattringer ${ }^{\dagger}$ and C.B. Lang \\ Institut für Theoretische Physik, \\ Universität Graz, A-8010 Graz, AUSTRIA
}

May 1992

\begin{abstract}
We study a simple 2-d model representing two fields with different mass and a 3 -point coupling term. The phase shift in the resonating 2-particle channel is determined from the energy spectrum obtained in Monte Carlo simulations on finite lattices. Masses and wave function renormalization constants of the fields as well as mass and width of the resonance are determined and discussed. The representation of scattering states in terms of the considered operators is analysed.
\end{abstract}

*Supported by Fonds zur Förderung der Wissenschaftlichen Forschung in Österreich, project $\mathrm{P} 7849$.

${ }^{\dagger}$ Present address: Max Planck Institut für Physik und Astrophysik, Föhringer Ring 6, D-8000 Munich, Germany 


\section{Introduction and motivation}

Determination of scattering phase shifts is still considered one of the open problems in the nonperturbative lattice approach to quantum field theory. Up to now bulk quantities, masses and matrix elements were the central objects. Scattering processes involve at least four asymptotic states; in QCD each of them is a bound state in terms of the original fields, indeed a very demanding situation. However, various contributions to this question exist [1]]-[10].

The problem is particularly pressing, as most of the hadronic states to be analysed are resonances and hence not asymptotic states of the field theory. This makes any rigorous control of finite size effects, which necessarily turn up due to numerical limitations, extremely difficult. Furthermore, phase shifts provide the only direct comparison to experiment. Resonance parameters are inferred from phase shifts whereas positions of resonance poles in the unphysical sheet are plagued by necessary assumptions implicit in the phenomenological analysis.

In a series of papers Lüscher [1]-[3] introduced a method to obtain scattering phase shifts from an analysis of the energy spectrum in a finite size system. Eventually this method should be used in the full 4-d theory [5], but before we can hope to attack the determination of phase shifts in lattice QCD it is appropriate to deal with simpler models. Lüscher and Wolff [4] studied the $\mathrm{O}(3)$ non-linear sigma model in $\mathrm{d}=2$, where analytic results for the scattering phases exist. That model may be interpreted as describing scattering of three mass-degenerate particles with certain symmetries. In order to discuss a situation of two fields with different masses and potentially resonating scattering behaviour we attacked another simple 2-d model [10]. Here we present a full account of our analysis and results.

The organisation of this paper is as follows. In the next section we briefly review Lüscher's approach for the calculation of scattering phases in a two dimensional lattice field theory. We also discuss the idea for the numerical determination of the scattering spectrum from Monte Carlo data as presented in [4]. Based on this, we formulate a method that allows one to compute the contribution of a lattice operator to physical states. In the third section we present our model and discuss basic properties and our Monte Carlo simulation. The fourth section contains results for the scattering parameters. A determination of the wavefunction renormalization constants provides bet- 
ter understanding of the observed dependence of the resonance width on the bare 3-point coupling. This is followed by a section where we present our results for the contributions of the lattice operators to the scattering states. We end with some concluding remarks.

\section{Determination of scattering parameters}

\subsection{Energy spectrum and phase shift}

Let us start with a brief review of Lüscher's approach [1]-[1]. Throughout the paper we consider the dimensional quantities as given in units of the appropriate powers of the lattice spacing $a$. Consider the scattering of two bosons of mass $m$ in a two dimensional system of finite spatial extension $L$, but infinite time extension. In this finite volume the momentum of a particle can only have discrete values. Assume that the interaction region is localized and smaller than $L$. Imposing periodic boundary conditions, the quantization condition for the relative momentum of two scattering particles reads

$$
2 \delta\left(k_{n}\right)+k_{n} L=2 n \pi, \quad n \in \mathbb{N},
$$

where $\delta(k)$ denotes the phase shift acquired in the interaction region. If its functional form is known, one could use this relation to find the quantized values $k_{n}$. On the other hand, given the momentum spectrum, (1) allows the determination of $\delta(k)$ for each $k$. This approach may be realized by utilizing the dispersion relation for the energy of two particles (of equal mass $m$ ) with relative momentum $k$

$$
W=2 \sqrt{m^{2}+k^{2}} .
$$

The energy spectrum in the two particle sector may be computed in a Monte Carlo simulation for a lattice with spatial extension $L$. Indeed, for given $L$ one measures a set of discrete values $W_{n}$, thus $k_{n}$ and with (1) the corresponding value $\delta\left(k_{n}\right)$. Repeating this procedure for various lattice sizes it is possible to find the phase shift for a large set of momentum values.

The power of this approach lies in the utilization of finite size effects for the determination of physical quantities. One will have to respect carefully the limitations. The interaction region and the single particle correlation length ought to be smaller than the spatial volume, in particular $1 / m<<$ 
L. Polarization effects due to virtual particles running around the torus should be under control. Lattice artifacts will turn up in $O\left(a^{2}\right)$ corrections, i.e. for large values of $k$. For the determination of the energy spectrum one should consider correlation functions of a sufficiently large number of observables with the correct quantum numbers, capable to represent the space of scattering states [1].

\subsection{Correlation functions}

We compute the connected cross-correlations

$$
M_{n m}(t):=\left\langle N_{n}{ }^{*}(0) N_{m}(t)\right\rangle_{c}=\left\langle N_{n}{ }^{*}(0) N_{m}(t)\right\rangle-\left\langle N_{n}{ }^{*}\right\rangle\left\langle N_{m}\right\rangle
$$

of operators in the scattering sector. Each $N_{n}, n=1,2 \ldots$ is defined on a single time slice, and $t$ denotes the separation of the time slices. Ideally we have to consider a complete set of states. The explicit form of the operators will be given in section 4, after introduction of the model.

The transfer matrix formalism (see e.g. [11]) yields the spectral decomposition

$$
M_{n m}(t)=\sum_{l=1}^{\infty} v_{n}^{(l)^{*}} v_{m}^{(l)} e^{-t W_{l}} .
$$

For simplicity we assume non-degenerate $W_{l}$, ordered increasingly. The amplitudes $v_{n}^{(l)}=\left\langle l \mid N_{n} 0\right\rangle$ are the projections of the states $\left|N_{n} 0\right\rangle$ (generated by the operators $N_{n}$ out of the vacuum) on the energy eigenstates $\langle l|$ of the scattering problem.

For the determination of the energy spectrum from (4) we follow 四. On a finite lattice, in the elastic regime below the four particle threshold (or the three particle threshold, depending on the model) there is a finite number of energy eigenstates. Only two-particle states contribute and the projections onto physical single particle states vanish. Also, in a Monte Carlo calculation one can only take into account a finite number $r$ of operators, which, however should exceed the considered number of eigenstates.

One can expect that the spectral decomposition of $M(t)$ is approximated rather well by the truncated matrix

$$
C_{n m}(t)=\sum_{l=1}^{r} v_{n}^{(l) *} v_{m}^{(l)} e^{-t W_{l}}, \quad n, m=1,2 \ldots r .
$$


The generalized eigenvalue problem

$$
C(t) \boldsymbol{\zeta}^{(k)}(t)=\lambda^{(k)}\left(t, t_{0}\right) C\left(t_{0}\right) \boldsymbol{\zeta}^{(k)}(t), \quad k=1,2, . ., r
$$

allows one to find the energy eigenvalues $W_{l}$ efficiently even for not too large values of $t$. In (6) we assume $t_{0}<t$, e.g. $t_{0}=1$. We require the $N_{n}, n=1,2, . ., r$ to be linearly independent, thus $C\left(t_{0}\right)$ is regular and the generalized eigenvalue problem is well defined. Indeed, it may be transformed to a standard eigenvalue problem for the matrix

$$
C^{-\frac{1}{2}}\left(t_{0}\right) C(t) C^{-\frac{1}{2}}\left(t_{0}\right),
$$

with identical eigenvalues $\lambda^{(k)}\left(t, t_{0}\right)$ and eigenvectors $\mathbf{u}^{(k)}(t)=C^{\frac{1}{2}}\left(t_{0}\right) \boldsymbol{\zeta}^{(k)}(t)$.

The solution for the eigenvalues is

$$
\lambda^{(k)}\left(t, t_{0}\right)=e^{-\left(t-t_{0}\right) W_{k}} \quad .
$$

This can be easily verified by inserting it into (6), leading to

$$
B^{(k)}(t) \zeta^{(k)}(t)=0
$$

where

$$
B_{n m}^{(k)}(t)=\sum_{l \neq k} v_{n}^{(l) *}\left[v_{m}^{(l)}\left(e^{-t W_{l}}-e^{-t_{0} W_{l}} e^{-\left(t-t_{0}\right) W_{k}}\right)\right] .
$$

Each row of the matrix $B(t)$ is a linear combination of the $r-1$ linearly independent vectors $\mathbf{v}^{(l)}, l=1,2, . ., k-1, k+1, . ., r$. Thus the space of the solutions of (9) has dimension 1 , as is needed to construct a normalized eigenvector for the corresponding standard eigenvalue problem. The effect of the truncation was shown to behave like $\exp \left[-\left(t-t_{0}\right) W_{r+1}\right]$ by a perturbation calculation [4].

To summarize, the determination of the spectrum in the scattering sector consists of the following steps. Compute the correlation matrix $M(t)$ for various values of $t$ and calculate $M^{-\frac{1}{2}}\left(t_{0}\right) M(t) M^{-\frac{1}{2}}\left(t_{0}\right)$. The dimension $r$ of $M$ cannot be chosen too large, because then the inversion turns out to be numerically unstable. Finally solve the eigenvalue problem for this matrix, and determine the spectrum from the exponential decay (8). 


\subsection{Eigenstates and spectral density}

Like in any Quantum Mechanics application the central problem is to consider a set of operators with a sufficient overlap with the energy eigenstates. We now demonstrate that the eigenvectors $\boldsymbol{\zeta}^{(k)}$ of the generalized eigenvalue problem contain information about the representation of the physical states by the chosen operators $N_{n}$.

Rewriting (9) gives

$$
\sum_{l \neq k} \mathbf{v}^{(l) \dagger}\left[\left(e^{-t W_{l}}-e^{-t_{0} W_{l}} e^{-\left(t-t_{0}\right) W_{k}}\right) \mathbf{v}^{(l)} \cdot \boldsymbol{\zeta}^{(k)}(t)\right]=0
$$

for all $k$. Since the $\mathbf{v}^{(l) \dagger}$ are linearly independent, their coefficients have to vanish, yielding $\mathbf{v}^{(l)} \cdot \boldsymbol{\zeta}^{(k)}(t)=0 \forall l \neq k$. Thus $\boldsymbol{\zeta}^{(k)}(t) \propto \mathbf{v}^{(k)}$ and, when normalized to unit length, independent of $t$. Introducing the normalized eigenvector $\boldsymbol{\zeta}^{(k)}=\mathbf{v}^{(k)} /\left|\mathbf{v}^{(k)}\right|$ also the eigenvectors $\mathbf{u}^{(k)}=C^{\frac{1}{2}}\left(t_{0}\right) \boldsymbol{\zeta}^{(k)}$ of the associated standard eigenvalue problem are time independent.

We may express the original approximation (5) to $M(t)$ in terms of the obtained eigenvectors,

$$
C_{n m}(t)=\sum_{l=1}^{r} \zeta_{n}^{(l)} \zeta_{m}^{(l)}\left|\mathbf{v}^{(l)}\right|^{2} e^{-t W_{l}}, \quad n, m=1,2 \ldots r .
$$

In particular consider the diagonal element

$$
C_{n n}(t)=\sum_{l=1}^{r}\left|\zeta_{n}^{(l)}\right|^{2}\left|\mathbf{v}^{(l)}\right|^{2} e^{-t W_{l}} .
$$

For a continuous energy spectrum,

$$
C_{n n}(t)=\int d W \rho_{n}(W) e^{-t W},
$$

and we identify $\left|\zeta_{n}^{(l)}\right|^{2}\left|\mathbf{v}^{(l)}\right|^{2} \propto \rho_{n}\left(W_{l}\right)$, the spectral density of the correlation function of operator $N_{n}$. We therefore find that $\left|\zeta_{n}^{(l)}\right|^{2}$ indicates the relative weight of the contribution of operator $N_{n}$ to the energy eigenstate $|l\rangle$.

In the calculation again one has to deal with the correlation matrix $M$ and not with the truncated form $C$. This implies that results obtained from $\boldsymbol{\zeta}^{(l)}:=M^{-\frac{1}{2}}\left(t_{0}\right) \mathbf{u}^{(l)}$, where $\mathbf{u}^{(l)}$ are the eigenvectors of $M^{-\frac{1}{2}}\left(t_{0}\right) M(t) M^{-\frac{1}{2}}\left(t_{0}\right)$, are affected by a term $O\left(e^{-t W_{r+1}}\right)$, where $r$ is the rank of $M$.

If energy eigenvalues are degenerate, the procedure does not apply and the amplitudes cannot be identified in a unique way. The method then gives equal importance for the involved operators. 


\section{Model and Monte Carlo simulation}

\subsection{Model}

We choose a model, which describes two light particles $\varphi$ that couple to a heavier particle $\eta$ giving rise to resonating behaviour. For this we couple two Ising fields through a 3 -point term. The action is given by

$$
\begin{aligned}
S= & -\kappa_{\varphi} \sum_{x \in \Lambda, \mu=1,2} \varphi_{x} \varphi_{x+\hat{\mu}} \\
& -\kappa_{\eta} \sum_{x \in \Lambda, \mu=1,2} \eta_{x} \eta_{x+\hat{\mu}} \\
& +\frac{g}{2} \sum_{x \in \Lambda, \mu=1,2} \eta_{x} \varphi_{x}\left(\varphi_{x-\hat{\mu}}+\varphi_{x+\hat{\mu}}\right) .
\end{aligned}
$$

Here, $\hat{\mu}$ denotes the unit vector in direction $\mu$. The values of the fields are restricted to $\{+1,-1\}$. Quantization corresponds to taking expectation values with the measure $Z^{-1} \exp (-S) d \varphi d \eta$ where $Z$ denotes the normalizing partition function. The sum runs over all sites $\left(x_{0}, x_{1}\right)$ of the euclidean $L \times T$ lattice $\Lambda \subset \mathbb{Z}_{L} \times \mathbb{Z}_{T}$ and we imply periodic boundary conditions. The 3 -point term was introduced in a nonlocal but symmetric way, because $\varphi_{x}^{2} \equiv 1$.

The case $g=0$ corresponds to the situation of two independent Ising models, each with a 2 nd order phase transition at $\kappa_{\text {crit }}=\frac{1}{2} \ln (1+\sqrt{2}) \simeq$ 0.44068. In the scaling limit the model describes an interacting boson with mass $m=-\log (\tanh \kappa)-2 \kappa$ [11]. Reformulating the Ising model at the phase transition as a theory of non-interacting fermions, it was shown that the scattering matrix assumes the value -1 independent of the momentum [12].

In the coupled case $(g>0)$, if we identify $\eta$ and $\varphi$ with particle states, the term proportional to $g$ gives rise to transitions like $\eta \rightarrow \varphi \varphi$ rendering $\eta$ a resonance in the $\varphi$ channel, when kinematically allowed. The notion of mass is then no longer well defined for the $\eta$ as it is no longer an asymptotic state of the theory. For us, this is just the situation of interest. Henceforth, whenever we say "mass" of the $\eta$ field we really mean the position where the phase shift assumes its resonating value. For given $g$, "masses" of the fields $\eta$ and $\varphi$ may be adjusted by calibrating the hopping parameters $\kappa_{\varphi}$ and $\kappa_{\eta}$. 


\subsection{Phase structure}

In the following we give some simple arguments about the phase structure of our model in the $\left(\kappa_{\varphi}, \kappa_{\eta}, g\right)$ space (in the thermodynamic limit).

For $g=0$ we have the Ising phase transitions for $\kappa_{\varphi}=\kappa_{\text {crit }}$ and $\kappa_{\eta}=\kappa_{\text {crit }}$. In the general case the model is even in the $\varphi$ field. In the symmetric phase of the $\eta$-field there is a symmetry $g \leftrightarrow-g$.

For $g>0$ we first consider the situation with a quenched $\eta$ configuration and discuss the dynamics of $\varphi$. The action then has just a hopping term for the $\varphi$-field with a locally varying coupling

$$
\omega_{x, \mu}=\kappa_{\varphi}-\frac{1}{2} g\left(\eta_{x}+\eta_{x+\hat{\mu}}\right) .
$$

For sufficiently small $g$ this is still a ferromagnetic system and the Griffiths inequalities apply. Thus all link expectation values are monotonically increasing with $\omega_{x, \mu}$. For sufficiently large $\kappa_{\varphi}$ there will be an ordered and for sufficiently small $\kappa_{\varphi}$ a disordered phase. This situation (Potts model with quenched random bonds) has been recently studied in detail [13, 14] where the authors prove that there is a second order phase transition. To our knowledge it is unclear, whether this behaviour survives for a dynamical $\eta$ field.

In order to examine the $\eta$ dynamics let us discuss two limiting cases. For $\kappa_{\varphi}=\infty$ the $\varphi$ spins are strictly ordered. Then the three-point term in (15) effectively acts like an external magnetic field of strength $g$. For non-zero $g$ the field $\eta$ has long range order and does not undergo a phase transition. On the other hand for $\kappa_{\varphi}=0, \varphi$ is completely disordered and the interaction term acts like an external random field for the $\eta$-spins. Random magnetic fields are expected to lower the effective dimensionality (i.e. raise the critical dimension by 2) [15]- [17]. Recently it was proved [13] that for a quenched random magnetic field the field $\eta$ remains disordered for all values of the hopping parameter. Hence we conclude that there is no phase transition in the $\eta$ field for finite $\kappa_{\eta}$ and $\kappa_{\varphi}=0, g>0$.

We expect therefore that the $\eta$ phase transition extending at $g=0$ over all values of $\kappa_{\varphi}$ disappears at $g>0$, at least for the edges $\kappa_{\varphi}=0, \infty$. Our numerical results for masses and bulk quantities show that the phase transition becomes weaker for $g>0$, changing into a cross-over like behaviour with large, but finite correlation length at the $\eta$-transition. 
To summarize these arguments, one cannot be sure that for $g \neq 0$ the model (15) undergoes a second order phase transition for both fields. This would indicate that the cutoff cannot be removed completely for $g>0$ and that there is no continuum limit possible, in which both fields describe mutually interacting physical particles with finite mass. We are always dealing with an effective model.

However, we are not presenting a model for a possible continuum field theory. All we want is to study the possibility of extracting phase shifts in a situation where two particles of different mass have an effective interaction. For this aim the lattice is considered more a technical means to approximate an underlying continuum model than a method for a rigorous construction of a continuum limit. In the range of bare couplings considered the model serves our purpose. However, not all combinations of correlation lengths (i.e. mass values) can be realized. In particular a study of lattice artifacts is not possible for arbitrary small lattice spacing.

\subsection{Cluster algorithm}

For the Monte Carlo simulation of Ising models the cluster algorithm [18] is a very powerful tool, both for generating new configurations and for defining and measuring improved estimators [19]. In our case with a 3-point term we have to modify it. We update the $\eta$ and the $\varphi$ field alternately.

Updating the $\eta$ spins: Bonds between neighboured spins of equal sign are kept with the probability $1-\exp \left(-2 \kappa_{\eta}\right)$. After identification of the connected clusters all clusters are flipped, each with separately determined probability

$$
\begin{aligned}
p_{\eta}^{\text {flip }} & =1 /\left(1+e^{-2 \alpha(C)}\right) \\
\alpha(C) & =\frac{g}{2} \sum_{x \in C, \mu=1,2} \eta_{x} \varphi_{x}\left(\varphi_{x-\hat{\mu}}+\varphi_{x+\hat{\mu}}\right) .
\end{aligned}
$$

This method has been suggested [18] in order to incorporate the effect of constant external fields.

Updating the $\varphi$ spins: This part of the action is treated like an Ising system with the locally varying hopping parameter $\omega_{x, \mu}$ introduced in (16). In identifying the clusters we keep bonds between like-sign neighbours alive with probability $1-\exp \left(-2 \omega_{x, \mu}\right)$ and flip clusters with probability $\frac{1}{2}$. 
The cluster method is ergodic and fulfills the detailed balance condition; for the values of $g$ considered it is a clear improvement over the Metropolis updating.

We study the model at three values of the 3-point coupling $g=0,0.02$ and 0.04; for later reference we denote these cases by I, II and III. Our first step was to tune the hopping parameters. We measured single particle propagators to adjust the $\varphi$-mass and find a crude estimate for the energy of the $\eta$ resonance. We tried to establish the values $m_{\varphi} \simeq 0.19$ and $m_{\eta} \simeq 0.5$. Table 1 gives the couplings where we performed systematic simulations on various lattice sizes.

Throughout this work we use $T=100$; the spatial extension $L$ varies between 12 and 60 . For each set of couplings and lattice size we performed typically $2 \times 10^{5}$ measurements. For the statistical errors we display one standard deviation, estimated with the Jackknife method (For a discussion see e.g. 20]).

\section{Results for the scattering parameters}

For the determination of the energy spectrum a precise knowledge of the single particle mass and related finite size effects is important. In this section we first discuss our results for the single particle state, then for the two particle channel, in particular the phase shifts. Finally we perform a com-

putation of wavefunction renormalization constants, to clarify the scaling of the resonance width with $g$.

\subsection{Single particle states}

Let us first study the single particle propagator. The operator of a $\varphi$ state with momentum

$$
p_{1, \nu}=2 \pi \nu / L, \quad \nu=-L / 2+1, \ldots, L / 2
$$

may be represented through

$$
\frac{1}{L} \sum_{x_{1} \in \Lambda_{x_{0}}} \varphi_{x_{0}, x_{1}} \exp \left(i x_{1} p_{1, \nu}\right)
$$


where $\Lambda_{x_{0}}$ denotes a timeslice of $\Lambda$. Its connected correlation function over temporal distance $t$ decays exponentially $\propto \exp \left(-p_{0, \nu} t\right)$ defining the single particle energy $p_{0, \nu}$; in particular we have $p_{0, \nu=0}=m_{\varphi}$.

The observed mass, as compared to the "real" mass at vanishing lattice spacing and infinite volume, incorporates contributions from polarization due to self interaction around the torus (decreasing exponentially with L) and lattice artifacts due to the finite ultra-violet cutoff (polynomial in the lattice constant a).

In fig. 1 we plot $p_{0, \nu}$, obtained for the pure Ising model (I) and $L=50$. We compare the values with the continuum spectral relation ,

$$
p_{0, \nu}=\sqrt{m^{2}+p_{1, \nu}^{2}}
$$

and find deviations $O\left((a p)^{2}\right)$, as expected 四. We also plot the dispersion relation for the lattice propagator of a Gaussian particle with mass $m$,

$$
p_{0, \nu}=\operatorname{arcosh}\left(1-\cos p_{1, \nu}+\cosh m\right),
$$

and find excellent agreement with the measured values. Thus this particular single particle state shows little deviation from the free lattice form. This results holds for models (II) and (III) too.

The Ising model may be considered the limit of a $\phi^{4}$-theory with infinite bare 4-point coupling $\lambda$. We are in the symmetric phase. Thus we expect the leading interaction to be due to an effective 4-point term. The finiteness of the spatial volume allows self-interaction with one particle running around the torus. This leads to a finite volume correction to the particle mass. Performing a perturbation calculation for two dimensional $\varphi^{4}$ theory along the lines of [21] one finds a decrease

$$
M(L)-m \propto \lambda L^{-\frac{1}{2}} e^{-m L}+O\left(\lambda^{2}, L^{-\frac{1}{2}} e^{-2 m L}\right) \quad,
$$

where $M(L)$ is the mass measured on a lattice of spatial size $L$, and $m$ is the infinite volume mass. A 3-point interaction leads to a different exponential.

Fig. 2, again for model (I), gives our results for $\ln [\sqrt{L}(M(L)-m)]$ vs. $L$ and indicates perfect agreement with (22). Indeed the fit to a linear behaviour gives a slope of $-1.047(31) \mathrm{m}$ for case (I) and $-1.015(25) \mathrm{m}$ for (III), in good agreement with the expected value $-m$. For situation (I) this is obvious, because we are in the symmetric phase where there is no 
3 -point coupling. In cases (II) and (III) the $\eta$ particle that enters the threepoint interaction is much heavier than the $\varphi$ and its contribution is therefore suppressed. This mass shift on finite lattices has also been confirmed in Monte Carlo simulations of the Ising model in 4 dimensions 22].

As already discussed in section 2, the infinite volume mass is explicitly known in the Ising model. We compare the measured value at $L=50$ for $g=0$ with this exact result and find a shift of $+0.00006(13)$. We use this shift to estimate the infinite volume mass values for the light particles in the coupled cases by subtracting it from the values measured at $L=50$, correspondingly (Anyway this shift lies within the errors).

Equivalencing the Ising system with an effective field theory of renormalized fields, the real valued variables $\Phi$ with the canonical continuum form of the kinetic and the mass term for bosons, transform to the Ising spins $\phi$ via $\Phi=\sqrt{\kappa} \phi$. The momentum space propagator for the $\Phi$ fields is

$$
G(p)=\frac{Z}{m_{r}^{2}+p^{2}} \quad \text { for } \quad p \longrightarrow 0
$$

where $m_{r}$ is the renormalized $\Phi$ mass and $Z$ the wave function renormalization constant. It may be determined from the susceptibility

$$
Z=\kappa m^{2} \frac{1}{L T} \sum_{x 1, x 2 \in \Lambda}\left\langle\phi_{x_{1}} \phi_{x_{2}}\right\rangle_{c}
$$

The renormalized field is given through $\Phi_{r}=\Phi / \sqrt{Z}=\sqrt{\kappa} \phi / \sqrt{Z}$.

Since the definitions for $\eta$ and $\varphi$ are identical, the symbol $\phi$ stands for either spin variable $\eta$ or $\varphi$. We use the high temperature results 23. for the susceptibility in the Ising model, to check the correctness of our numerical results for $Z$ in the uncoupled case.

Only $Z_{\varphi}$ is well defined, since only $\varphi$ is an asymptotic state of the full theory. However, in the discussion of our results we want to compare the obtained value for the resonance width with the tree-level predictions of an effective Lagrangian model; for this we need to consider properly renormalized effective fields. Only for the sake of this qualitatively interesting comparison we determine an "effective" w.f. renormalization constant $Z_{\eta}$, aware of the ill-definedness of that quantity in case (II) and (III). 


\subsection{Two particle sector}

\subsubsection{Operators and phase shifts}

In the 2-particle channel we consider operators with total zero momentum and quantum numbers of the $\eta$,

$$
\begin{aligned}
N_{1}\left(x_{0}\right)= & \frac{1}{L} \sum_{x_{1} \in \Lambda_{x_{0}}} \eta_{x_{1}, x_{0}}, \\
N_{j}\left(x_{0}\right)= & \frac{1}{L^{2}} \sum_{x_{1}, y_{1} \in \Lambda_{x_{0}}} e^{i p_{j}\left(x_{1}-y_{1}\right)} \varphi_{x_{1}, x_{0}} \varphi_{y_{1}, x_{0}}, \\
& \text { with } p_{j}=\frac{2 \pi(j-2)}{L}, \quad j=2,3, \ldots
\end{aligned}
$$

and measure all cross-correlations (3). The operators $N_{j>1}$ describe two $\varphi$-particles in the CM system with relative momentum $p_{j}$. Due to the interaction they do not correspond to eigenstates of our model. Indeed they are eigenstates of the Gaussian model of free bosons. However, if the set is complete, the diagonalization of section 2 provides the necessary information on the energy spectrum of this channel.

The number of operators considered in (3) should be chosen larger than the number of states in the elastic regime $2 m_{\varphi} \leq W<4 m_{\varphi}$ and not larger than $L / 2$ to be linearly independent. A larger set provides a better representation of the eigenstates but enhances the numerical noise. We work with between 4 and 6 operators depending on $L$ (Only as a testing case we once consider 8 operators for a particular run at $L=60, g=0.02)$. For the product $M^{-\frac{1}{2}}\left(t_{0}\right) M(t) M^{-\frac{1}{2}}\left(t_{0}\right)$. we choose $t_{0}=1$; for our statistics, usually the diagonalization becomes unstable around $t=t_{\max } \simeq 5-8$, depending on $L$. The values $W_{\alpha}$ are determined by averaging $\log \left[\lambda_{\alpha}(t) / \lambda_{\alpha}(t+1)\right]$ for $1<t<t_{\max }$. For each lattice size we determine the low lying part of the energy spectrum. Each value corresponds, via the spectral condition (2), to a value of $k$ and with (1) to a phase shift $\delta(k)$.

For $g=0$ we have the situation of two independent Ising systems. As discussed above, the critical Ising model in $\mathrm{d}=2$ has an S-matrix equal to -1 [12], and we therefore expect a phase shift $\delta_{\text {Ising }}=-\frac{\pi}{2} \quad(\bmod \pi)$ in the scaling regime. In fig. 3 we exhibit the energy spectrum (a) for a system of non-interacting Gaussian fields (curves due to (1) with phase shift $\delta=0$ ) and (b) as obtained in our simulation of the Ising model. The curves for 
the two particle states in fig. 3b are due to (四) for $\delta=-\frac{\pi}{2}$ and we find excellent agreement. The single particle state $\eta$ (horizontal line at $W=$ $0.5)$ is completely decoupled from the $\varphi \varphi$ states and we therefore observe degeneracies in the energy levels at certain values of $L$. Fig. 4 gives the results for the phase shift $\delta(k)$ determined from the data for the Ising model (fig. 3b) as a function of the dimensionless momentum $k / m_{\varphi}$.

Already here we identify two sources for the relative magnitude of the error bars. Small values of $k$ result from values of $W$ near $2 m_{\varphi}$, at large $L$. Close to this threshold, due to the functional dependence of (2) the statistical error in $W$ transforms into a relatively larger error of $k$ and thus $\delta(k)$. Higher $k$, on the other hand, stem from the large values of the energy, where the statistical fluctuations are intrinsically larger.

Figs. 5a and $5 \mathrm{~b}$ exhibit results for $g \neq 0$. For most of the points the errors are smaller than the symbols, typically $0.5 \%$ of the energy value. The degeneracies in the energy spectrum have now disappeared, resulting in avoided level crossing with a gap that grows with $g$. The corresponding phase shift data are given in fig.6.

One should keep in mind that points at neighboured values of $k$ may come from quite different values of $L$ and different branches of energy levels. In order to emphasize this situation we have chosen corresponding symbols to denote energy values on one branch, i.e. for one value of $n$. Each branch, from small to large $L$ contributes to the whole range of $k$ values; it starts at larger values of $W$ (or $k$ ) with a phase shift close to $\pi / 2$, passes through the resonance value at the plateau and approaches $-\pi / 2$ towards smaller $k$. The overall consistent behaviour is impressive.

The high energy values, corresponding to large $k$ may feel the lattice cutoff effects $O\left(a^{2}\right)$ as well as possible mis-representation of the energy eigenstates by the considered operators. We discuss the second problem in a subsequent section. Within the accuracy of our data none of these effects seems to be a problem.

\subsubsection{Resonance parameters}

The observed overall behaviour has a simple interpretation. A resonance may be parametrized with the effective range approximation

$$
\frac{k}{W} \cot \delta_{\operatorname{Res}}=a-b k^{2}
$$


At the resonance $\delta_{\text {Res }} \simeq \frac{\pi}{2}+\frac{W-m_{R}}{\Gamma_{R} / 2}$, or

$$
m_{R}=2 \sqrt{\left(m_{\varphi}\right)^{2}+(a / b)}, \quad \Gamma_{R}=\frac{4}{b m_{R}^{2}} \sqrt{\frac{a}{b}} .
$$

Adding this resonance phase to the Ising model background phase the spectrum in the resonating channel should approximately obey

$$
\begin{aligned}
\delta_{\text {total }}(k) & \equiv \delta_{\text {Ising }}+\delta_{\text {Res }}(k)=-\arctan \frac{a-b k^{2}}{k / W} \\
W & =2 \sqrt{\left(m_{\varphi}\right)^{2}+\left(-\frac{2}{L} \delta_{\text {total }}(k)+\frac{2 n \pi}{L}\right)^{2}}, n=0,1, \ldots
\end{aligned}
$$

Here $n$ denotes the various energy levels in relation (1). We superimpose this theoretically expected behaviour of the spectrum on the measured points in figs. 3-6.

The parameters $m_{R} \equiv m_{\eta}$ and $\Gamma_{R} \equiv \Gamma_{\eta}$ may be determined from a direct fit to the shoulders in the energy curves. However, this fit turns out to be not very stable and has much larger statistical errors than a determination from the resulting phase shifts as discussed below. We fit the values of $\tan \delta$ in the region $-1.45<\delta<1.45$ to a straight line in $k^{2}$ according to (29). This gives the effective range parameters $a, b$ and our estimates for $m_{\eta}, \Gamma_{\eta}$ in table 1 . The resulting $\chi^{2}$ indicates that the statistical errors of our phase shift data and the errors of $W$ may be underestimated (cf. [10]).

The ratio of the resonance widths for (III) and (II) is smaller than the value 4 , the square of the ratio of the couplings $g$ that one naively expects from the Born approximation. In doing that, however, we should first write down an effective Lagrangian with properly renormalized effective fields $\eta_{r}$ and $\varphi_{r}$, with kinetic parts and a three-point term. For sake of such a comparison we have to determine the wave function renormalization constants $Z_{\varphi}$ and $Z_{\eta}$, although the second is ill-defined as mentioned earlier in sec. 4.1. From this information we may estimate an effective three-point coupling

$$
g_{\text {eff }}=g \frac{1}{\kappa_{\varphi} \sqrt{\kappa_{\eta}}} Z_{\varphi} \sqrt{Z_{\eta}},
$$

which may be a more suitable number to enter the tree-level formula.

With this naive procedure we obtain $Z_{\eta}, Z_{\varphi}$ and $g_{\text {eff }}$ for all three values of the bare coupling $g$ (see table 1 ). Both $Z_{\eta}$ and $Z_{\varphi}$ are smaller than 1 , even 
in the uncoupled case. These values decrease for increasing $g$. The values for $g_{\text {eff }}$ are $0.0568(6)$ and $0.0938(8)$ for $g=0.02$ and $g=0.04$ respectively. With these values, the ratio $\left(g_{\text {eff }}(0.04) / g_{\text {eff }}(0.02)\right)^{2}=2.73(11)$ is closer to the observed ratio $\Gamma_{\eta}(0.04) / \Gamma_{\eta}(0.02)=2.13(26)$. The naive argument based on tree-level perturbation theory appears to be at least qualitatively correct. However, for a better identification of our model with an effective field theory, one has to take into account further interaction terms of higher order in the fields. In fact the preferred definition of a renormalized 3-point coupling is just from the observed (physically well defined) width.

\section{Representation of the physical states}

Is the number of lattice operators considered for the determination of the energy levels in the scattering channel sufficiently large? To analyse the representation of the physical states by the considered operators (25) we compute the vectors $\boldsymbol{\zeta}^{(l)}=M^{-\frac{1}{2}}\left(t_{0}\right) \mathbf{u}^{(l)}$, normalized to unit length. Then $\left(\zeta_{n}^{(l)}\right)^{2}$ provides a measure on how much $N_{n}$ contributes to the physical eigenstate $|l\rangle$. As discussed in sect. 2.3 there should be no dependence on $t$. This is consistent with the numerical results and therefore we average the values for all available $t$.

Fig. 7 shows the relative weights $\left(\zeta_{n}^{(l)}\right)^{2}$ for $l=1,2,3$ and $n=1,2,3$ for case (III). Due to the normalization of $\boldsymbol{\zeta}^{(l)}$ the individual contributions can assume values between 0 and 1 . If the three displayed operators are sufficient to represent the considered state, their contributions should add up to 1 .

Since these values are displayed for all considered lattice sizes, a remark concerning the number $r$ of components in $\zeta^{(l)}$, which is equal the rank of $M$, is appropriate. For small $L$, the lattice is statistically "nervous", and the inversion of $M$ is possible only for a small number $r$ of operators (e.g. $r=4$ at $L=12$, while $r=8$ at $L=60$ ). At small $L$, for the lowest energy eigenstate $|1\rangle$ in the scattering sector mainly $N_{1}$ contributes (see the circles in fig. 7a). Only $\zeta_{1}^{(1)}$ is considerably different from zero. Hence for the lowest state $|1\rangle$ it makes no difference whether the vector $\boldsymbol{\zeta}^{(1)}$ is normalized at length 3 or 4 , because the 4th entry is very small anyway. For the higher states $|l\rangle$ more than four entries of $\boldsymbol{\zeta}^{(l)}$ may be necessary. However, due to the numerical instability of the inversion at small $L$, only four entries are available. Thus 
the results for the representation of the higher physical states appear shifted for small $L$. For larger $L$ this technical problem vanishes: The results for at least the three lowest eigenstates are reliable.

Compare the energy spectrum of fig. 5b with fig.7. Let us first discuss the lowest energy state $|1\rangle$, represented by circles in fig.5b, with respect to fig. 7a. For small $L, W_{1}$ is close to the resonance energy. There the state is completely dominated by the operator $N_{1}$ (circles in fig. 7a), which describes an $\eta$-particle at rest. For increasing $L, W_{1}$ decreases and approaches the 2-particle threshold. This manifests itself in a drastic decline of the contribution of $N_{1}$, accompanied by an increase of the amplitudes for two $\varphi$-particles at rest (operator $N_{2}$, triangles) and with relative unit-momentum (operator $N_{3}$, squares). For lattice size larger than 20, the energy $W_{1}$ is already too far below the resonance energy so that the energetically higher $\eta$ at rest cannot contribute much to $|1\rangle$. The state is built out of $N_{2}$ and $N_{3}$ (with energies as shown in fig. 3a) almost exclusively.

For the second lowest state $|2\rangle$ (fig. 7b) the situation is reversed. As seen in fig. 5b, for small $L$ the energy $W_{2}$ is high above the resonance energy, so the $\eta$-state $N_{1}$ (circles) does not contribute. The state is dominated by the lowest lying 2- $\varphi$ operators. For increasing $L, W_{2}$ decreases and as it crosses the resonance energy, the contribution of $N_{1}$ peaks. Leaving the resonanceplateau, state $|2\rangle$ is again dominated by the energetically favourable operator $N_{3}$. The contribution of $N_{4}$ is substantial as well, but not shown in the picture.

Fig. $7 \mathrm{c}$ gives the contributions to $|3\rangle$. In the considered $L$-region it does not show any dramatic behaviour. For $L<50$ the energy is too high for $N_{1}$ and $N_{2}$ contributions and the state is built out of $N_{3}$ and higher operators. Only for the largest $L$ the $\eta$-amplitude grows. For small $L$ the remark concerning the rank of the correlation matrix made earlier applies. Between $L=12$ and $L=16$ there is a discontinuity in the $N_{3}$ contributions, due to the discussed truncation effect. The diagonalization at $L=16$ includes one operator more than that at $L=12$. This operator has nonvanishing contributions and therefore affects the $N_{3}$ amplitude via the normalization of $\boldsymbol{\zeta}^{(3)}$.

In the case (I) of two uncoupled Ising models we get a similar picture. $N_{1}$ then measures the particle mass in the completely disconnected $\eta$ system and does not affect the $\varphi$ states and vice versa. The lowest eigenstates states of fig. $3 \mathrm{~b}$ turn out to be mixtures of the $\varphi \varphi$-states $N_{n}, n \geq 2$. 
In particular, it is easy to identify misrepresentation of energy eigenstates in terms of the considered operators. At higher energies one observes a shift of the weight factors $\left(\zeta_{n}^{(l)}\right)^{2}$ towards higher operators $N_{n}$. Fig. 8 gives an example of a situation, where the center of weight has moved to relatively high values of $n$. It is obvious, that more operators should be included for a good representation of that state.

Numerically we observe, that a truncation of the correlation matrix $M(t)$ leads to a rise of the higher energy levels. However, in the data presented here and used in the determination of the phase shift this effect is under control. For the low lying energies it is negligible and for the largest energy level it is still smaller than the statistical error.

\section{Concluding remarks}

In our Monte Carlo study we have established, that Lüscher's suggestion for determining phase shifts is indeed a very reliable method, at least in $\mathrm{d}=2$. Our simple model describes a system of two particles with a three-point coupling. The coupling constants have been chosen such that one of the states may become a resonance. Indeed we find resonating phase shifts in this channel. As a byproduct we also obtain the phase shift of a pure Ising model.

The following remark concerns just the particular model studied here: As discussed, for $g \neq 0$ we cannot expect to perform a continuum limit. Most likely it is not possible to increase the correlation length arbitrarily keeping the ratio $m_{\eta} / m_{\varphi}$ fixed. Numerically we observed, that the $\eta$-contribution to the peak in the specific heat becomes less pronounced for increasing values of $g$ (This is the technical reason for not studying even broader resonance widths). Therefore, in this model, it might not be possible to study lattice artifacts in a more systematic way.

The study of the representation of scattering states by the operators entering the correlation functions sheds light on the problem of constructing good approximations for the energy eigenstates. In particular it allows to control unwanted truncation effects.

For small $L$ one is confined to the first plateau only. Translating a $L$ independent constant energy $W=m_{\eta}$ into a phase shift we get a variety 
of $\delta$-values, all for a constant $k=\frac{1}{2} \sqrt{m_{\eta}^{2}-4 m_{\varphi}^{2}}$, just what we expect for a zero width resonance: a stable particle state. For a naive estimator of the resonance energy one therefore might consider just the single particle operator, ignoring the states of higher energy coming from above. However, increasing the lattice size would not improve the result (In fact, the resonance is no asymptotic state of the theory). There can be no avoided level crossing, as there are no 2-particle states. Thus there will be no information on the resonance width. On the other hand, ignoring the $\eta$-state and considering only $\varphi \varphi$ states, is insufficient, too. It is of crucial importance to include all operators (with the quantum numbers of that sector) with energies close to the considered energy eigenstates. For further discussions, in particular with regard to the situation in QCD see [5].

How can we understand the limit $g \rightarrow 0$ ? The energy gap becomes smaller and the level crossing region narrower. The phase shift approaches step function behaviour. In the Ising limit the phase shift is then compatible with a constant $(\bmod \pi)$.

Acknowledgment: We have benefitted much from discussions with $\mathrm{H}$. Gausterer, M. Lüscher, M. Salmhofer and E. Seiler, and we want to thank. 


\section{References}

[1] M. Lüscher, Commun. Math. Phys. 104 (1986) 177

[2] M. Lüscher, Commun. Math. Phys. 105 (1986) 153

[3] M. Lüscher, Nucl. Phys. B354 (1991) 531

[4] M. Lüscher, U. Wolff, Nucl. Phys. B339 (1990) 222

[5] M. Lüscher, Nucl. Phys. B364 (1991) 237

[6] U. Wiese, Nucl. Phys. B (Proc. Suppl.) 9 (1989) 609

[7] C. Michael, Nucl. Phys. B327 (1989) 515

[8] M. Guagnelli, E. Marinari and G. Parisi, Phys. Lett. B 240 (1990) 188

[9] T.A. DeGrand, Phys. Rev. D43 (1991) 2296

[10] C.R. Gattringer, C.B. Lang, Phys. Lett. B274 (1992) 95

[11] For details cf. e.g. C. Itzykson and J.-M. Drouffe, Statistical Field Theory (Cambridge University Press, Cambridge: 1989)

[12] M. Sato, T. Miwa and M. Jimbo, Proc. Japan Acad. 53, Ser.A (1977) 6; B. Berg, M. Karowski and P. Weisz, Phys. Rev. D19 (1979) 2477

[13] M. Aizenman and J.Wehr, Phys. Rev. Lett. 62 (1989) 2503; Commun. Math. Phys. 130 (1990) 489

[14] K. Hui and A.N. Berker, Phys. Rev. Lett. 62 (1989) 2507

[15] Y. Imry, S.-K. Ma, Phys. Rev. Lett. 35 (1975) 1399

[16] G. Parisi, N. Sourlas, Phys. Rev. Lett. 43 (1979) 744

[17] D. Andelman, H. Orland, L.C.R. Wijewardhana, Phys. Rev. Lett. 52 (1984) 145 
[18] R.H. Swendsen and J.-S. Wang, Phys. Rev. Lett. 58 (1987) 86; J.-S. Wang, Physica A161 (1989) 249

[19] M. Sweeny, Phys. Rev. B27 (1983) 4445; U. Wolff, Phys. Rev. Lett. 62 (1989) 361

[20] M.C.K. Yang, D.H. Robinson, Understanding and Learning Statistics by Computer, (World Scientific: 1986)

[21] M. Lüscher, in Fields, Strings and Critical Phenomena, E. Brézin and J. Zinn-Justin, ed.s, Les Houches, Session XLIX, 1988 (Elsevier, Amsterdam: 1989).

[22] I. Montvay, P. Weisz , Nucl. Phys. B290[FS20] (1987) 327; Ch. Frick, K. Jansen, J. Jersák, I. Montvay, P. Seuferling and G. Münster, Nucl. Phys. B331 (1990) 515

[23] M.F. Sykes, D.S. Gaunt, P.D. Roberts, J.A. Wyles, J. Phys. A 5 (1972) 624 


\section{Tables}

Table 1: This table summarizes the parameters for our simulations together with the measured values of masses and resonance width (in units of the lattice constant). We also show wave function renormalization constants and the effective 3-point couplings.

\begin{tabular}{|l|l|l|l|}
\hline Model & I & II & III \\
\hline$g$ & 0. & 0.02 & 0.04 \\
$\kappa_{\varphi}$ & 0.3948 & 0.3897 & 0.3700 \\
$\kappa_{\eta}$ & 0.3268 & 0.3323 & 0.3700 \\
\hline$m_{\varphi}$ & 0.189921 & $0.18965(13)$ & $0.19014(13)$ \\
$m_{\eta}$ & 0.499545 & $0.5008(4)$ & $0.5112(3)$ \\
$\Gamma_{\eta}$ & 0. & $0.0047(4)$ & $0.0100(3)$ \\
$Z_{\varphi}$ & $0.722(2)$ & $0.706(4)$ & $0.671(4)$ \\
$Z_{\eta}$ & $0.849(3)$ & $0.816(5)$ & $0.619(4)$ \\
$g_{\mathrm{eff}}$ & 0. & $0.0568(6)$ & $0.0938(8)$ \\
\hline
\end{tabular}




\section{Figures}

Fig. 1: The dispersion relation of our single particle propagator (circles, model (I) for $L=50$ ) approaches the continuum behaviour (broken curve) for small $p_{1, \nu}$; it follows nicely the lattice propagator of a free Gaussian particle (full curve).

Fig. 2: We plot our values for $\log [(M(L)-m) \sqrt{L}]$ vs. $L$, model (I); these results for the single particle mass follow the expected exponential dependence on the spatial size (22).

Fig. 3: (a) The 2-particle energy levels for a free Gaussian model $(\delta=0)$ as functions of the lattice size $L$. The dotted line indicates the energy of a stable $\eta$ with $m_{\eta}=0.5$. (b) The measured energy levels for the $\varphi \varphi$ and $\eta$ channel for the coupling constant values $g=0$ vs. the spatial lattice extension $L$. The full curve shows the theoretical expectations for the values from (1) for $\delta_{\text {Ising }}=-\frac{\pi}{2}$, the horizontal line denotes the $\eta$ mass level. The dashed lines indicate the 2- and the 4-particle thresholds.

Fig. 4: The results of the phase shift for model (I), i.e. corresponding to the energy data in fig. $3 \mathrm{~b}$, vs. $k / m_{\varphi}$. The full curve gives the theoretical expectation $\delta_{\text {Ising }}=-\frac{\pi}{2}$. The dashed line indicates the 4-particle threshold.

Fig. 5: The measured energy levels for the $\varphi \varphi$ channel for the coupling constant values (a) $g=0.02$ and (b) $g=0.04$, vs. the spatial lattice extension $L$. The full curves show the theoretical expectations for the values from (11) and $\delta_{\text {total }}$ as discussed in the text, eq.(30). The dashed lines indicate the 2 and the 4-particle thresholds.

Fig. 6: The phase shift as determined from the energy levels in figs. 5a and $5 \mathrm{~b}$ vs. $k / m_{\varphi}$; the full curves denote the theoretical expectations as discussed in the text. The dashed line indicates the 4-particle threshold.

Fig. 7: Contributions of the operators $N_{1}, N_{2}$ and $N_{3}$ (circles, triangles, squares) to the energy eigenstates (a) $|1\rangle$, (b) $|2\rangle$ and (c) $|3\rangle$, for case (III), displayed as a function of the lattice size $L$. 
Fig. 8: Contributions $\left(\zeta_{n}^{(6)}\right)^{2}$ of the considered operators $N_{n}$ to the energy eigenstate $|6\rangle$ at $L=60$ for model (II). 